\title{
On a Notion of Extensionality for Artifacts
}

\author{
Lech Polkowski $^{1}$ and Maria Semeniuk-Polkowska ${ }^{2}$ \\ Polish-Japanese Institute of Information Technology ${ }^{1}$. Koszykowa 86. 02008 \\ Warszawa, Poland \\ Chair of Formal Linguistics ${ }^{2}$. Warsaw University. Browarna 8/12. 00956 Warszawa \\ Poland \\ emails: polkow@pjwstk.edu.pl; m.polkowska@uw.edu.pl
}

\section{Abstract}

The notion of extensionality means in plain sense that properties of complex things can be expressed by means of their simple components, in particular, that two things are identical if and only if certain of their components or features are identical; e.g., the Leibniz Identitas Indiscernibilium Principle: two things are identical if each applicable to them operator yields the same result on either; or, extensionality for sets, viz., two sets are identiccal if and only if they consist of identical elements. In mereology, this property is expressed by the statement that two things are identical if their parts are the same. However, building a thing from parts may proceed in various ways and this unexpectedly yields various extensionality principles. Also, building a thing, may lead to things identical with respect to parts but distinct with respect, e.g., to usage. We address the question of extensionality for artifacts, i.e., things produced in some assembling or creative process and we formulate the extensionality principle for artifacts which takes into account the assembling process and requires for identity of two artifacts that assembling graphs for the two be isomorphic in a specified sense.

\section{Mereology in a Nutshell}

The primitive notion of mereology due to Leśniewski, cf., Leśniewski [8], [9], [10], Srzednicki et al. [18], is a notion of a part; for an in-depth, autoritative review of mereology, consult Simons [17]; also, consult Casati-Varzi [7] for a treatment of mereology from the point of view of spatial reasoning. Given some things in a collection $U$, a relation of a part is a binary relation $\pi$ on $U$ which is required to be

M1 Irreflexive: For each $x \in U$ it is not true that $\pi(x, x)$

M2 Transitive: For each triple $x, y, z$ of things in $U$, if $\pi(x, y)$ and $\pi(y, z)$, then $\pi(x, z)$

The relation of part induces the relation of an ingredient, ingr, due to Leśniewski [8] defined as

$$
\operatorname{ingr}(x, y) \Leftrightarrow \pi(x, y) \vee x=y
$$


Clearly,

Proposition 1. The relation of ingredient is a partial order on things.

We formulate the third axiom with a help from the notion of an ingredient.

M3 (Inference) For things $x, y$, the property

$I(x, y)$ : The property $O(x, y)$ : For each thing $t$, if ingr $(t, x)$, then there exist things $w, z$ such that ingr $(w, t), \operatorname{ingr}(w, z), \operatorname{ingr}(z, y)$

implies that $\operatorname{ingr}(x, y)$

The predicate of overlap, $O v$ in symbols, is defined by means of

$$
O v(x, y) \Leftrightarrow \exists z \cdot \operatorname{ingr}(z, x) \wedge \operatorname{ingr}(z, y)
$$

Using the overlap predicate, one can write the property $O(x, y)$ down in the form

Ov $(x, y):$ For each $t$ with ingr $(t, x)$, there exists $z$ such that ingr $(z, y)$ and $O v(t, z)$

The notion of a mereological class follows, cf. [8]: for a non-vacuous property $\Phi$ of things, the class of $\Phi$, denoted $C l s \Phi$ is defined by the conditions

C1 If $\Phi(x)$, then ingr $(x, C l s \Phi)$

$\mathrm{C} 2$ If ingr $(x, C l s \Phi)$, then there exists $z$ such that $\Phi(z)$ and $O v(x, z)$

In plain language, the class of $\Phi$ collects in an individual thing all objects satisfying the property $\Phi$.

The existence of classes is guaranteed by an axiom.

M4 For each non-vacuous property $\Phi$ there exists a class $C l s \Phi$

The uniqueness of the class follows.

Proposition 2. For each non-vacuous property $\Phi$, the class $C l s \Phi$ is unique.

Proof. Assuming that for some $\Phi$ there exist two distinct classes $Y_{1}, Y_{2}$, consider ingr $\left(t, Y_{1}\right)$. Then, by $\mathrm{C} 2$, and (2), there exists $z$ such that $O v(t, z)$ and $\operatorname{ingr}\left(z, Y_{2}\right)$. It follows by M3 that $\operatorname{ingr}\left(Y_{1}, Y_{2}\right)$. By symmetry, $\operatorname{ingr}\left(Y_{2}, Y_{1}\right)$ holds and Proposition 1(2) implies that $Y_{1}=Y_{2}$

\section{Extensionality for things from Mereology point of view}

In Leśniewski Mereology, extensionality is derivable from the axioms in the form: 
(EP) (Extensionality Principle) For things $x, y: x=y$ if and only if $x$ and $y$ have the same parts

Clearly, only the implication from right to left may need a proof. Assume then that $x$ and $y$ have the same parts. The identity $x=y$ follows from the

Proposition 3. Each thing $z$ is the class of all its ingredients.

Indeed, each part of $z$ is its ingredient (fulfilling $\mathrm{C} 1$ ) and for an ingredient $w$ of $z$ either $w=z$ or $\pi(w, z)$ in either case fulfilling obviously $\mathrm{C} 2$.

It turns out that extensionality may be defined in some other ways: Varzi [20] considers two more principles of extensionality, viz.,

(UC) (Uniqueness of Composition) For things $x, y: x==_{U} y$ if and only if $x$ and $y$ are classes of the same things in a collection $F$

(EC) (Extensionality of Composition) For things $x, y: x=_{E} y$ if and only if $x$ and $y$ are classes of the same collection $P$ of pairwise disjoint things

Varzi [20] gives a thorough analysis of those three principles, showing that they are not equivalent. This analysis may be recapitulated in a nutshell here for the benefit of the reader; first, both (EP) and (EC) are implied by (UC): assuming (UC) we admit (EP) by virtue of Proposition 3 and (EC) is a particular case of (UC).

But, (EP) implies neither (EC) nor (UC): that both implications fail was shown in Varzi [20] (cf. Fig. 1) with a simple example of disjoint atoms $a, b, c$ which induce $d=C l s\{a, b\}$ and $e=C l s\{b, c\}$ as well as $x=C l s\{d, c\}$ and $y=C l s\{a, e\}$; we have $x=C l s\{a, b, c\}, y=C l s\{a, b, c\}$, (EP) holds as distinct things have distinct collections of parts and (UC) and (EC) fail because $x$ and $y$ are classes of the same disjoint atoms $a, b, c$.

Existence of atoms is implied by the assumption of well-foundedness, cf., Aczel [1], Barwise and Moss [2].

(WFU) We say that the universe of things $U$ is $\pi$-well-founded if and only if there is in $U$ no decreasing $\pi$-sequence i.e. a sequence of things $\left\{x_{i}: i \in N\right\}$ such that $\pi\left(x_{i+1}, x_{i}\right)$ for each $i$

An atom in $U$ is a thing $x$ such that no $y \in U$ satisfies $\pi(y, x)$. It follows that being an atom in $U$ is an absolute notion, not depending on the thing the atom is a part of. $A t(x)$ denotes the property of being an atom and a part of $x$.

Under (WFU), the following hold.

Proposition 4. (WFU) implies

(1) each thing $x$ in $U$ contains an atom as an ingredient. 
(2) each thing $x$ in $U$ is the class of the property $A t(x)$.

(3) (EC) implies (UC), i.e., (EC) and (UC) are equivalent under (WFU).

For the proof, (1) is obvious; for (2), assume that, to the contrary, there is $x$ in $U$ which is not the class of $A t(x)$. By $\mathrm{C} 2$, there is an ingredient $y$ of $x$ disjoint (i.e. not overlapping) to each atom of $x$; but $y$ has an atom as an ingredient and this atom is as well an atom of $x$, a contradiction.

For (3) we may need a lemma which follows directly from the class definition $\mathrm{C} 1, \mathrm{C} 2$.

LEMMA. If a thing $x$ is the class of the property $\mathrm{F}$ and each $\mathrm{y}$ in $\mathrm{F}$ is the class of the property $\mathrm{P}(\mathrm{y})$, then $x$ is the class of the property $\bigvee_{y \in F} P(y)$.

We prove now (3). Assume that $x, y$ are classes of things satisfying the property F. For each $y$ in $F$, consider the property $A t(y)$; by $(2), y=C l s A t(y)$ for $y$ in $F$, hence by LEMMA, $x=C l s \bigvee_{y \in F} A t(y)$ and $y=C l s \bigvee_{y \in F} A t(y)$. As the collection $\bigvee_{y \in F} P(y)$ is pairwise disjoint, by (EC), $x=y$, satisfying (UC).

In general, as shown in Varzi [20] (cf. Fig. 2 therein), (EC) implies neither (EP) nor (UC); clearly, the example is possible only in a non-well-founded universe.

We have mentioned three types of extensionality immanent to composition of things from parts directly or via class forming. However, things are often composed of parts in systematic usage-oriented ways. Those things are called commonly artifacts ('made by art'), or, artefacts. Things composed of the same parts may have very distinct forms and properties, e.g., a robot built of parts supplied as NXT 2.0 may be a walking one or a crawling one, see [13]. This fact is to be somehow recorded in the description of an artifact as a thing obtained in a creative process.

\section{On the notion of an artifact}

The term artifact means, etymologically, a thing made by art, which covers a wide specter of things, from man-made things of everyday usage to abstract pieces of mathematical proofs, software modules, or concertos. All those distinct things are unified in a scheme dependent on some common ingredients in their making, cf., e.g., a concise discussion in SEP [16]. We cannot include here a discussion of vast literature on ontological, philosophical and technological aspects of this notion, we mention only a thorough analysis of ontological aspects of artifacts in Borgo and Vieu [4] in which authors propose also a scheme defining artifacts. It follows from discussion by many authors that important in analysis of artifacts are such aspects as: authorship, intended functionality, parthood relations. Analysis of artifacts is closely tied to design and assembly, cf., Boothroyd [5] and Boothroyd, Dewhurst and Knight [6] as well as Salustri [14] and Seibt [15]. A discussion of mereology with respect to its role in domain science and engineering and computer science can be found in Bjoerner [3] and Polkowski [12]. 
We thank the anonymous referee for turning our attention to a book by Zdzisław Pawlak [11] in which the author develops a theory of manufacturing processes modeled on the mechanical assembly process of a thing from parts along a scheme adopted as a tree. Though no mereology is mentioned, yet the author defines parts of things as leaves of assembling trees (calling them details) for those things and derives basic mereological properties of parts in this setting.

We attempt at a definition of an artifact as a thing obtained over a collection of things as a most complex thing in the sense of not being a part of any thing in the collection; to aspects of authorship (operator)and functionality, we add a temporal aspect. We propose a number of requirements governing the assembling process. We also regard a parallel process of design as an assembling process.

\subsection{A definition of an artifact as a design or assembly product}

We single out: a category of operators $P$, a category of functionalities $F$, a linear time $T$ with the time origin 0 ; the process of artifact design/synthesis will be carried out by designers from the category $D$ and assemblers from the category $A$. The domain of things is a category Things $(D, A, P, F, \pi)$ of things endowed with a part relation $\pi$ of which we do assume $\pi$-well-foundedness. The assignment operator $S$ acts as a partial mapping on the Cartesian product $D \times A \times \operatorname{Things}(D, A, P, F, \pi)$ with values in the category Tree of trees.

For some things $x$ in $\operatorname{Things}(D, A, P, F, \pi)$ and some pairs $(d, a) \in D \times A$, the operator $S$ assigns a unique tree $S(d, a)(x)=\operatorname{Tree}(d, a)(x)$ which is the design/synthesis tree for the pair $(d, a)$ and the thing $x$. Its root node is representing the thing $x$ designed by $d$, with assembly tools designed by $a$, and produced by some operators in $P$. Each node $w$ of the tree $\operatorname{Tree}(d, a)(x)$ is the root of the tree of the form $\operatorname{Tree}(d, a)(y)$ for some thing $y$ which does represent the design/assembling scheme for $y$.

The replacement relation $\sim$ is defined on the category $\operatorname{Things}(D, A, P, F, \pi)$ by means of

$$
x \sim y \Leftrightarrow \exists z \pi(x, z) \wedge \exists z . \pi(y, z) \wedge[\pi(x, z) \Leftrightarrow \pi(y, z)] \text { for each thing } \mathrm{z}
$$

Classes of $\sim$ are categories of replaceable things. The category of $x$ is denoted as $\operatorname{Cat}(x)$. From (3) it follows that $x \sim y$ implies that neither of $x, y$ is a part of the other. We define a predicate $\pi_{d, a}$ on the domain of $\sim ; \pi_{d, a}(y)$ means that the thing $y$ is a part of some thing in the universe Things $(D, A, P, F, \pi)$.

The process of assembling will be formally described by means of the predicate

$$
\operatorname{Art}\left(d, a, p,<x_{1}, \ldots, x_{k(y)}>, y, f, t, \operatorname{Tree}(d, a)(y)\right)
$$

with $p$ in $P, f$ in $F, t$ in $T$, which reads an assembler a projects an assembly scheme according to the design $d$ which yields from things $x_{1}, \ldots, x_{k(y)}$ the thing $y$ of functionality $f$ at the time $t$ according to the scheme Tree $(d, a)(y)$ with an operator $p$. The predicate $\operatorname{Asmbl}(x, i, y, p, f, t)$ reads the thing $x$ is used 
in the position $i$ in assembling the thing $y$ of functionality $f$ at some time $t$ and with some operator $p$. We propose the following axioms of assembling. The tuple $<d, a, p,<x_{1}, \ldots, x_{k(y)}>y, f, t, \operatorname{Tree}(d, a)(y)>$ is the signature of $y$ when $\operatorname{Art}\left(d, a, p,<x_{1}, \ldots, x_{k(y)}>, y, f, t, \operatorname{Tree}(d, a)(y)\right)$ holds.

Art0. For each thing $x$, each node of the tree $\operatorname{Tree}(d, a)(x)$ is labeled with a label of the form

$$
\left(d, a, p,<z_{1}, \ldots, z_{k(y)}>, y, f, t, \operatorname{Tree}(d, a)(y)\right)
$$

with $\operatorname{Tree}(d, a)(y)$ a subtree of $\operatorname{Tree}(d, a)(x)$, and $t^{\prime}<t$.

Art 1.

$$
\begin{gathered}
\operatorname{Art}\left(p,<x_{1}, \ldots, x_{k(y)}>, y, f, t, \operatorname{Tree}(d, a)(y)\right) \\
\wedge \\
\forall i \leq k(y) \cdot \operatorname{Art}\left(p_{i},<z_{1}, \ldots, z_{k\left(x_{i}\right)}>, x_{i}, f_{i}, t_{i}, \operatorname{Tree}(d, a)\left(x_{i}\right)\right) \\
\Rightarrow \forall i \leq k(y) \cdot p_{i} \subset p, f \subset f_{i}, t_{i}^{\prime}<t
\end{gathered}
$$

The relation $p^{\prime} \subset p$ is meant as: if $p^{\prime}$ is allowed to assemble a thing $z$ then $p$ is allowed to assemble $z$ (a more complex operator has a wider scope); the relation $f \subset f^{\prime}$ means if $y$ is usable in assembling $z$ then $x_{i}$ is usable in assembling $z$ (a less complex thing has a wider usage); the inequality $t_{i}<t$ means that less complex $x_{i}$ is assembled before $y$ is assembled.

Art 2. $\operatorname{Asmbl}\left(x_{i}, i, y, p, f, t\right) \wedge \operatorname{Cat}\left(x_{i}\right)=\operatorname{Cat}(z) \Rightarrow \operatorname{Asmbl}(z, i, y, p, f, t)$.

Art 3. $\operatorname{Art}\left(p,<x_{1}, \ldots, x_{k(y)}>, y, f, t, \operatorname{Tree}(d, a)(y)\right) \wedge \operatorname{Cat}(y)=\operatorname{Cat}\left(y^{\prime}\right)$ $\Rightarrow \operatorname{Art}\left(p,<x_{1}, \ldots, x_{k(y)}>, y^{\prime}, f, t, \operatorname{Tree}\left(y^{\prime}\right)\right)$.

Things of the same category are interchangeable.

Art 4. $\operatorname{Art}\left(p,<x_{1}, \ldots, x_{k(y)}>, y, f, t, \operatorname{Tree}(d, a)(y)\right) \Rightarrow \pi\left(x_{i}, y\right)$ for $i \leq k(y)$.

Each thing is assembled from its parts.

Art 5. $\pi(y, x) \Rightarrow$ there exists a node $w$ in $\operatorname{Tree}(d, a)(x)$ with the signature of the form $\left(d, a, p,<z_{1}, \ldots, z_{k(w)}>, w, \operatorname{Tree}(d, a)(w)\right)$ such that $\operatorname{Cat}(w)=\operatorname{Cat}(y)$.

Each part of the thing $x$ up to its category is to be used in the assembling of $x$ at some appropriate step of the assembling process.

Art 6. Each leaf of each tree of the form Tree $(d, a)($.$) is of the signature$ form $(d, a, p, a, f, t,\{a\})$ with $a$ an atom.

Initial assembling begins with elementary parts. 
Art 7.

$$
\begin{gathered}
\forall i . \operatorname{Cat}\left(x_{i}\right)=\operatorname{Cat}\left(z_{i}\right) \wedge \operatorname{Art}\left(p,<x_{1}, \ldots, x_{k(y)}>, y, f, t, \operatorname{Tree}(y)\right) \\
\wedge \\
\operatorname{Art}\left(p^{\prime},<z_{1}, \ldots, z_{k(y)}>, y^{\prime}, f^{\prime}, t^{\prime}, \operatorname{Tree}\left(y^{\prime}\right)\right) \\
\wedge \pi_{d, a}(y) \wedge \pi_{d, a}\left(y^{\prime}\right) \Rightarrow \operatorname{Cat}(y)=\operatorname{Cat}\left(y^{\prime}\right) .
\end{gathered}
$$

Assembling factorizes through categories.

Art 8. Formulas

$$
\operatorname{Art}\left(d, a, p,<z_{1}, \ldots, z_{k(y)}>, y, f, t, \operatorname{Tree}(y)\right)
$$

and

$$
\operatorname{Art}\left(d^{\prime}, a^{\prime}, p^{\prime},<z_{1}, \ldots, z_{k\left(y^{\prime}\right)}>, y^{\prime}, f^{\prime}, t^{\prime}, \operatorname{Tree}\left(y^{\prime}\right)\right)
$$

are regarded as equivalent if and only if their signatures are identical, $k(y)=$ $k\left(y^{\prime}\right), \operatorname{Cat}\left(z_{i}\right)=\operatorname{Cat}\left(z_{i}^{\prime}\right)$ for $i \leq k(y), \operatorname{Tree}(d, a)(y)$ and $\operatorname{Tree}(d, a)\left(y^{\prime}\right)$ are isomorphic as unlabeled trees.

The label $\operatorname{Art}\left(d, a, p,<z_{1}, \ldots, z_{k(y)}>, y, f, t, \operatorname{Tree}(y)\right)$ will be called the label at the node $y$.

Art 9. Trees Tree $(d, a)(x)$, Tree $(d, a)(y)$ are identical if and only if they are isomorphic as unlabelled trees and signatures at all corresponding nodes of $x$ and $y$ are equivalent in the sense of Art 8 .

Art 10. $\neg \exists w, p, f, t, i . A \operatorname{smbl}(y, i, w, p, f, t) \Rightarrow y$ in $\operatorname{ARTIFACTS}(D, A, P, F, \pi)$.

The category ARTIFACTS $(D, A, P, F, \pi)$ consists of 'final' things.

Art 11. (EA) (Extensionality for artifacts) Two things, in particular, artifacts, $x$ and $y$ are identical if and only if trees $\operatorname{Tree}(d, a)(x), \operatorname{Tree}(d, a)(y)$ are identical.

Art 12. Each non-artifact thing may be used in synthesis of only one other thing.

Corollary 1. $y$ in ARTIFACTS $\Rightarrow \neg \exists z . \pi(y, z)$.

We may construct the Ontology Graph $G_{O G}$. Its vertex set $V_{O G}$ is the set of categories of things and the edge set $E_{O G}$ consists of all pairs $\left(\operatorname{Cat}\left(x_{i}\right), \operatorname{Cat}(y)\right)$ for all cases $\operatorname{Art}\left(p,<x_{1}, \ldots, x_{k(y)}>, y, f, t\right)$ which hold. From Art 1 - Art 12 it 
follows that $G_{O G}$ is a forest.

Art 11 is the Extensionality for Artifacts Principle implying that two artifacts are identical if and only if their synthesis trees are isomorphic, i.e. they are composed of replaceable things under same designer, assembler, and operator, at the same time. Functionalities and timing are identical as well.

Corollary 2. By Art4-6, Art8, Art12, for each artifact $x$, the tree Tree $(d, a)(x)$ is uniquely determined by its atoms $A t(x)$ and $x=\operatorname{Cls} A t(x)$.

We allow some modifications in definitions of properties (EP), (UC), (EC), viz., in those definitions, we replace the term "parts" in (EP) with the phrase "parts of the same category", and in (UC), (EC) we replace phrases, respectively, "things in a collection F", "pairwise disjoint things" with, respectively, phrases "things of the same category in a collection F", " pairwise disjoint things of the same category".

Corollary 3. In our setting for artifacts, assuming the identity as defined by Art11, (EC), (UC) and (EP) in modified versions are equivalent.

\section{Conclusion}

Artifacts have been defined here as things obtained in a process determined by postulates Art $0-$ Art 12 over a well-founded collection of things. Modified by factoring through the equivalence Cat identity postulates (EP), (EC), (UC), shown to be non-equivalent in general by Varzi, are shown to be equivalent when the identity is understood in the sense of Art 11.

\section{References}

1. Aczel, P. (1988): Non-Well-Founded Sets. CSLI Publications. Stanford.

2. Barwise, J.; Moss, L. (1996): Vicious Circles: On the Mathematics of Non-WellFounded Phenomena. CSLI Publications. Stanford.

3. Bjoerner, D. (2012-13): A role for mereology in domain science and engineering. In: Calosi, C.; Graziani, P. (eds.)(2012): Mereology and the Sciences, Springer Synthese Library, to appear.

4. Borgo, S.; Vieu, L.(2009): Artefacts in formal ontology. In: Meijers, A. (ed.): Handbook of Philosophy of Technology and Engineering Sciences. Elsevier, pp. 273-308.

5. Boothroyd, G.(2005): Assembly Automation and Product Design (2nd ed.). Taylor and Francis, Boca Raton FL.

6. Boothroyd, G.; Dewhurst, P.; Knight, W.(2002): Product Design for Manufacture and Assembly (2nd ed.). Marcel Dekker, New York.

7. Casati, R.; Varzi, A. C.(1999): Parts and Places. MIT Press, Cambridge MA.

8. Leśniewski, S. (1916): Podstawy Ogólnej Teoryi Mnogości, I (Foundations of General Set Theory, I, in Polish). Prace Polskiego Koła Naukowego w Moskwie, Sekcya Matematyczno-przyrodnicza, No. 2, Moscow. 
9. Leśniewski, S. (1927-1931): O podstawach matematyki (On foundations of mathematics, in Polish). (1927) Przeglạd Filozoficzny XXX, pp 164-206; (1928) Przegląd Filozoficzny XXXI, pp 261-291; (1929) Przegląd Filozoficzny XXXII, pp 60-101; (1930) Przeglạd Filozoficzny XXXIII, pp 77-105 (1930); (1931) Przegląd Filozoficzny XXXIV, pp 142-170.

10. Leśniewski, S. (1982): On the foundations of mathematics. Topoi 2, pp 7-52.

11. Pawlak, Z. (1969): Mathematical Aspects of the Production Process (in Polish: Matematyczne Aspekty Procesu Produkcyjnego). The State Economic Publishers, Warszawa, Poland.

12. Polkowski, L. (2012-13): Mereology in engineering and computer science. In: Calosi, C.; Graziani, P. (eds.)(2012): Mereology and the Sciences, Springer Synthese Library, to appear.

13. http://www.tuvie.com/wp-content/uploads/lego-mindstorms-nxt-2.0-robots4.jpg

14. Salustri, F. A.(2002): Mereotopology for product modelling. A new framework for product modelling based on logic. J. Design Res. 2.

15. Seibt, J.(2009): Forms of emergent interaction in general process theory. Synthese 1666, pp. 479-512.

16. SEP (Stanford Encyclopedia of Philosophy): Artifact; available http://plato. stanford. edu/entries/artifact

17. Simons, P. (1987): Parts: A Study in Ontology. Clarendon, Oxford.

18. Srzednicki, J., Surma, S. J., Barnett, D., Rickey, V. F. (eds.) (1992): Collected Works of Stanisław Leśniewski. Kluwer, Dordrecht.

19. Thomasson, A.(2007): Artifacts and human concepts. In: Margolis, E.; Laurence, S. (eds.): Creations of the Mind: Theories of Artifacts and Their Representation. Oxford University Press, pp. 52-73.

20. Varzi, A. C. (2008): The extensionality of parthood and composition. The Philosophical Quarterly 58 pp. 108-133. 